munication). They present typically as macropapular erythematous rashes, though dermatographism, nausea, vertigo, and malaise have also been reported. The reactions commonly develop several hours after administration of the radiopharmaceutical and may last up to 48 hours. These localised reactions are considered to be minor, and only one other severe systemic reaction has been described; in that case rechallenge by skin testing proved a causal relation. ${ }^{2}$ We thought that rechallenge was unwise in our case given the severity of the reaction.

Diphosphonates are present in several radiopharmaceuticals other than those used for bone scanning; examples are agents for labelling red cells (Amerscan Stannous Agent, Amersham International) and imaging the liver (Microlite, Du Pont (United Kingdom)). Alternative agents should thus be used for patients known or suspected to be sensitive to diphosphonates; bone scanning may be done with pyrophosphates. In addition, hypersensitivity reactions may occur in patients with Paget's disease being treated with therapeutic concentrations of diphosphonates. The radiopharmaceutical that we injected contained only $0.5 \mathrm{mg}$ diphosphonate, whereas single therapeutic doses are in the range $15-30 \mathrm{mg}^{3+}$ and are administered by intravenous infusion over less than two hours. We therefore suggest that skin testing for sensitivity be considered when pharmaceuticals containing diphosphonates are used therapeutically and it would not compromise management of the patient.

1 Keeling D, Sampson CB. Adverse reactions to radiopharmaceuticals: incidence, reporting, symptoms, treatment. Nuklearmedizin 1986;23 (suppl):478-82.

2 Ramos-Gabatin A, Orzel JA, Maloney TR, Murnane JE, Borchert RD. Severe systemic reaction to diphosphonate bone imaging agents: skin testing to predict allergic response and a safe alternative agent. $\mathcal{F}$ Nucl Med 1986;27: $1432-5$.

3 Ralston SH, Alzaid AA, Gardner MD, Boyle IT. Treatment of cancer associated hypercalcaemia with combined aminohydroxypropylidene diphosphonate hypercalcaemia with combined aminohyd

4 Cardwell B, Harris AL. Single high dose aminohydroxypropylidene diphos phonate infusions to treat cancer-associated hypercalcaemia. Lancet 1986; $165-6$.

(Accepted 15 April 1988)
The General Infirmary at Leeds, Leeds LS1 3EX M Larvin, FRCS, pancreatic research fellow

M R J Lansdown, FRCS, lecturer in surgery

M J McMahon, FRCS, senior lecturer, university department of surgery

A G Chalmers, FRCR, consultant radiologist J H Turney, MRCP, consultant physician

A M Brownjohn, FRCP, consultant physician, regional renal unit

Correspondence to: $\mathrm{Mr}$ McMahon.

\section{Plasmapheresis: a rational treatment for fulminant acute pancreatitis?}

\section{Larvin, M R J Lansdown, M J McMahon, A G Chalmers, J H Turney, A M Brownjohn}

Roughly $5-10 \%$ of attacks of acute pancreatitis prove fatal. Death usually results from fulminant multiorgan failure, with activation of complement, kinin, fibrinolytic, and coagulation cascades, which may be triggered by pancreatic proteases liberated into the circulation. $\alpha_{2}$ Macroglobulin, functionally the most important plasma antiprotease, binds proteases irreversibly, forming a complex of around 800000 daltons. The active site of the protease remains free, so that the complexes retain the ability to hydrolyse peptides. Conformational changes in $\alpha_{2}$ macroglobulin trigger phagocytosis by the reticuloendothelial system, clearing complexes from the circulation with a half life of about 10 minutes in health. We recently showed that clearance is impaired in severe pancreatitis,' possibly because the reticuloendothelial system is overwhelmed in a manner similar to that which occurs in severe sepsis.

Plasmapheresis is a logical means to aid the reticuloendothelial system and we describe its use in two patients with fulminant acute pancreatitis.

\section{Case reports}

Case 1-A 68 year old man developed respiratory and renal failure 72 hours after admission to hospital with severe gall stone pancreatitis (Ranson score 8). Ventilation and haemodialysis were begun and the pulmonary capillary wedge pressure monitored. CT angiography showed peripancreatic necrosis. Four days after admission he developed severe fever, tachycardia, hypotension, and peripheral vasodilatation. $\mathrm{He}$ continued to deteriorate despite antibiotics and plasmapheresis was performed through femoral arterial and venous cannulas. A $2 \cdot 8$ litre exchange was completed with a Curesis plasma separator $\left(3 \times 10^{6}\right.$ dalton filter; Organon Teknika, Cambridge, United Kingdom), replacing filtrate with an equivalent volume of fresh frozen plasma. Vital signs stabilised and the fever decreased (figure). The APACHE II score, which summates abnormalities in 12 physiological variables, ${ }^{3}$. fell from 33 to 27 . Five days later he deteriorated again, and CT guided aspiration yielded infected peripancreatic debris. Debridement was carried out at laparotomy but failed to influence progressive multiorgan failure, and the patient died 18 days after admission.

Case 2-A 33 year old man was transferred to this hospital four days after admission elsewhere for severe acute alcoholic pancreatitis (Ranson score 6). ${ }^{2} \mathrm{He}$ developed a severe toxic confusional state and gross hypoxia $\left(\mathrm{PaO}_{2} 4 \cdot 7 \mathrm{kPa} ; \mathrm{F}_{\mathrm{I}} \mathrm{O}_{2}\right.$ air $)$. CT angiography
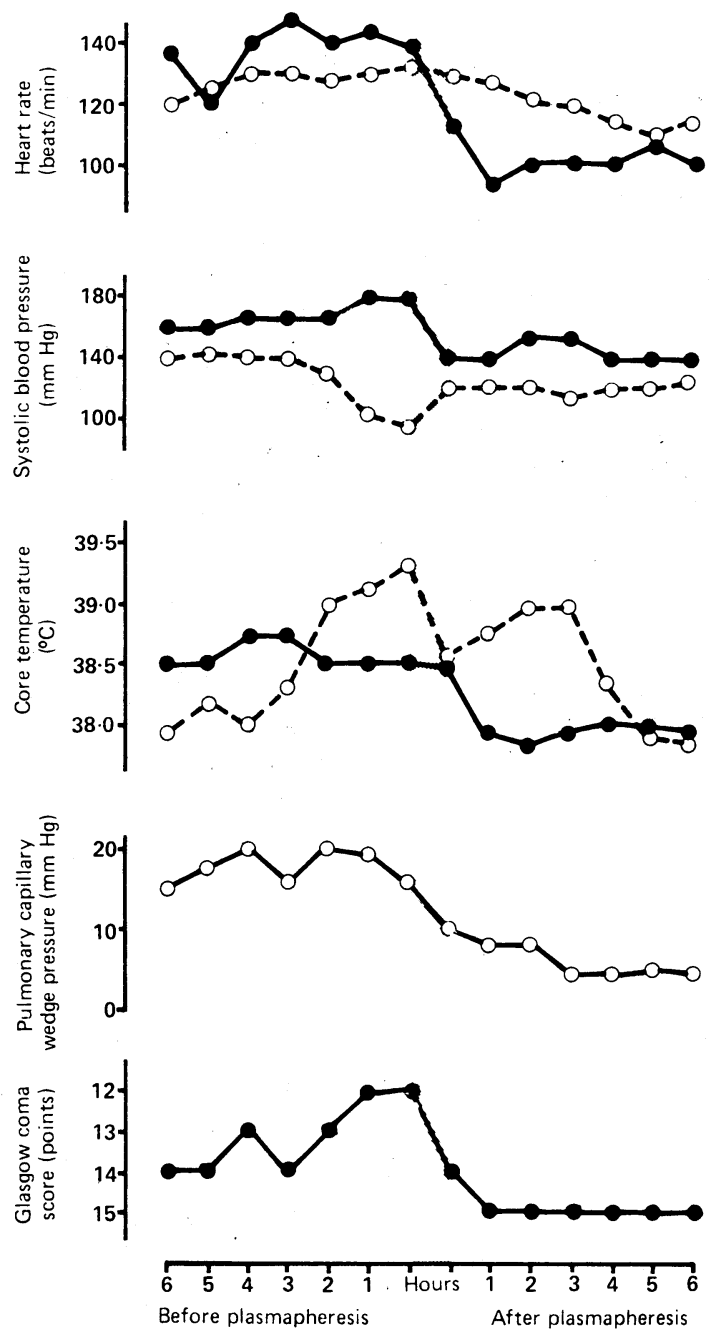

Observations before and after plasmapheresis in cases $1(\mathrm{O}-\mathrm{O})$ and 2 ( 
showed necrosis of the pancreatic body and tail. On the eighth day of the attack he became hypertensive, unconscious, and unresponsive, with increasing fever despite antibiotics. A 1.8 litre plasma exchange was performed as in case 1. Confusion cleared rapidly, vital signs and oxygenation improved, fever decreased (figure), and the APACHE II score fell from 17 to 9. Next day the necrotic pancreatic tail and body were debrided at laparotomy and irrigating drains inserted. He made a complete recovery and was discharged 38 days later.

\section{Comment}

Many patients with fulminant acute pancreatitis have pancreatic necrosis, and survival depends on debridement of necrotic or infected tissue. If operation is undertaken during the initial week survival is unlikely, ${ }^{+}$and the outlook is similarly bleak if multiorgan failure becomes established.' There is a logical role for treatments that allow debridement to be undertaken at a stage when recovery can be expected. Plasmapheresis scavenges various circulating toxins, including protease- $\alpha_{2}$ macroglobulin complexes. Our findings are preliminary, but the similarity of response was impressive, and in both cases there was a large reduction in the APACHE II score with the expectation of an increased probability of survival. Plasmapheresis appeared to induce more pronounced changes in the course of acute pancreatitis than other treatments that we have employed. Further evaluation is needed, preferably in patients who have not developed the manifestations of overt multiorgan failure and in the setting of a protocol which includes surgical debridement, when appropriate, on the basis of objective criteria including CT angiography and precise physiological monitoring.

1 Larvin M, Switala SF, McMahon MJ. Impaired clearance of circulating protease-antiprotease complexes during acute pancreatitis: an importan proteasc Sniprome

2 Ranson JH, Rifkind KM, Roses DF, Eng K, Spencer FC. Prognostic signs and the role of operative management in acute pancreatitis. Surg Gynecol Obste 1974:139:69-81.

3 Knaus WA, Draper EA, Wagner DP, Zimmermann JE. APACHE-II: a severity of disease classification system. Crit Care Med 1985;13:818-29.

+ Smadja C, Bismuth H. Pancreatic debridement in acute necrotising pancreatitis an obsolete procedure? Br $\mathcal{F}$ Surg 1986;73:408-10.

5 Glazer G, Dudley HAF. The surgical management of pancreatic abscess. Ann $R$ Coll Surg Engl 1987;69:249-50.

(Acçpted 9 Mư 1988)

\section{Human leucocyte antigen in a Chinese family with thyrotoxic periodic paralysis in Singapore}

\section{F Sum, A C K Fok, K T Tan, S H Chan, J S Cheah}

University Department of Medicine, Singapore General Hospital, Singapore 0316, Republic of Singapore CF Sum, MRCP, registrar A C K Fok, MRCP, registrar K T Tan, MRCP, registrar J S Cheah, FRACP, professor of medicine

\section{Department of}

Microbiology, National University of Singapore and World Health Organisation Immunology Centre, Singapore S H Chan, FRCPA, professor of microbiology

Correspondence and requests for reprints to: DrSum.
Periodic paralysis is a dramatic complication in some patients who have thyrotoxicosis (Graves' disease). It occurs more commonly in thyrotoxic men of Mongoloid descent.

The familial occurrence of thyrotoxic periodic paralysis is, however, infrequent. To date there have been only a few reports of familial thyrotoxic periodic paralysis. Most reports have been based on a positive family history elicited during questioning of the patient. We report the first, to our knowledge, documented occurrence of familial thyrotoxic periodic paralysis in a Chinese family in Singapore together with the human leucocyte antigen genotypes of the family members.

\section{Case report}

A 31 year old Chinese man was admitted with characteristic clinical and biochemical features of hypokalaemic thyrotoxic periodic paralysis. His thyrotrophin receptor antibody concentration was

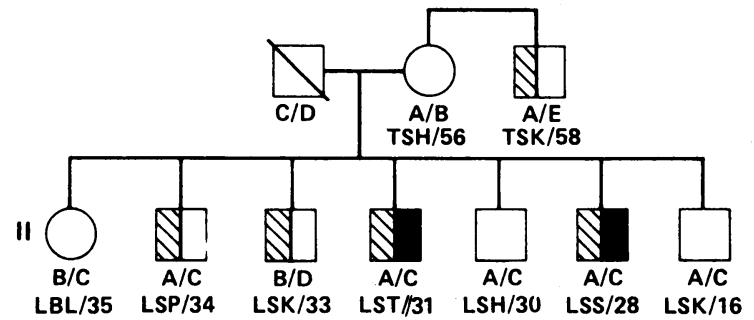

Human leucocyte antigen haplotypes:

$A=A 9 \quad$ BW22 DR9 DRw53 DQw3

$B=A 2 \quad \cdots . . . \quad$ DR9 DRw53 DOw3

$C=A 11 \quad B 16 \quad$ DR2 $\quad$ DRw52 DOw

$D=A 26 \quad B 15$ DR5 DRw52 DQw1

$\mathrm{E}=\mathrm{A} 11$ BW46 DR2 ….. DQW1

Family pedigree and human leucocyte antigen genotypes
$195 \mathrm{IU} / 1$ (normal < $10 \mathrm{IU} / \mathrm{l})$. He was treated with oral potassium supplements together with propranolol and carbimazole. The next day he had completely regained his muscular power. The case records of all his immediate family members who had thyrotoxicosis or thyrotoxic periodic paralysis, including a thyrotoxic maternal uncle, were recalled and studied. Subsequently the human leucocyte antigen type of all the immediate family members and maternal uncle was tested with the National Institutes of Health lymphocyte microcytotoxicity method.' The figure shows the pedigree and human leucocyte antigen genotypes of the family.

\section{Comment}

Lately there has been much interest in the genetics of thyrotoxicosis and thyrotoxic periodic paralysis. Chan et al reported an association between human leucocyte antigen Bw46 and DR9 and thyrotoxicosis in Chinese patients. ${ }^{2}$ Those members of the family whom we studied who had documented thyrotoxicosis (with or without periodic paralysis) were haplotype A or B, each of which contains the alleles DR9, DRw53, and DQw3.

In addition, Yeo et al showed that the haplotypes A2 Bw22 and Aw19 B17 were associated with thyrotoxic periodic paralysis among Chinese patients in Singapore. ${ }^{3}$ It was reported that Bw22 and B17 were in linkage disequilibrium with $\mathrm{A} 2$ and Aw19, respectively. In another study Cheah concluded that it was the alleles Bw22 and B17 that carried an increased relative risk for thyrotoxic periodic paralysis. ${ }^{4} \mathrm{He}$ also observed that the haplotype A2 Bw22 occurred only in Chinese thyrotoxic patients who had periodic paralysis and was absent in Chinese thyrotoxic patients who did not have periodic paralysis.

In the family that we studied, besides the two siblings who had thyrotoxic periodic paralysis, three other siblings were haplotype $A$ thus carrying the allele Bw22. It is tempting to speculate that thyrotoxic periodic paralysis may occur in these three siblings should the thyrotoxic state recur.

Familial thyrotoxicosis occurs more commonly than familial thyrotoxic periodic paralysis. Several studies of human leucocyte antigen have been performed in such families. On reviewing the cumulative results of studies of white and Chinese subjects Tian et al noted that of 36 different families that had multiple cases of 